\title{
Pharmacological Study of Sildenafil on Healthy Volunteers
}

\section{Eliwa HA $^{1^{*}}$, Eldin AA $^{2}$ and Salah MM}

${ }^{1}$ Department of Pharmacology and Toxicology, College of Pharmaceutical Sciences and Drug Manufacturing, MISR University for Science and Technology (MUST), Giza Egypt

${ }^{2}$ Department of Pharmaceutics and Industrial Pharmacy, College of Pharmaceutical Sciences and Drug Manufacturing, MISR University for Science and Technology (MUST), Giza Egypt

${ }^{3}$ Department of Bioequivalence, MAKIN Research Center (MRC), Egypt

"Corresponding author: Eliwa HA, Department of Pharmacology and Toxicology, College of Pharmaceutical Sciences and Drug Manufacturing, MISR University for Science and Technology (MUST), Giza Egypt, E-mail: dr.heshamab@gmail.com

Received date: October 09, 2018; Accepted date: November 12, 2018; Published date: November 17, 2018

Copyright: $@ 2018$ Eliwa HA, et al. This is an open-access article distributed under the terms of the Creative Commons Attribution License, which permits unrestricted use, distribution, and reproduction in any medium, provided the original author and source are credited.

\begin{abstract}
The present study is a comparative single-dose, open-label, randomized, three-treatment, six-sequence, threeperiod, crossover, in vivo study to determine the bioequivalence of two test products: Test 1; Sildenamax (Sildenafil $100 \mathrm{mg}$ ), Test 2; Satenafil (Sildenafil $100 \mathrm{mg}$ ) manufactured by Organo for Pharmaceutical and Chemical Industries, versus VIAGRA ${ }^{\circledR}$ (Sildenafil $100 \mathrm{mg}$ ) manufactured by Pfizer Egypt, USA and its Subsidiary in UK after a single oral dose administration given to healthy adult volunteers under fasting conditions. The subjects who conform to the study entry criteria were dosed according to a randomization schedule. Furthermore, the study was designed and completed according to the good clinical and laboratory practices
\end{abstract}

Keywords: Pharmacokinetic parameters $\left(\mathrm{C}_{\max }, \mathrm{T}_{\max }, \mathrm{K}_{\mathrm{e}}, \mathrm{AUC}_{0-\mathrm{t}}\right.$, and $\mathrm{AUC}_{0-\infty}$ ); Sildenafil

\section{Introduction}

Sildenafil inhibits the cGMP-specific phosphodiesterase type 5 (PDE5) which is responsible for degradation of cGMP in the corpus cavernosum located around the penis. Penile erection during sexual stimulation is caused by increased penile blood flow resulting from the relaxation of penile arteries and corpus cavernosal smooth muscle. This response is mediated by the release of nitric oxide (NO) from nerve terminals and endothelial cells, which stimulates the synthesis of cGMP in smooth muscle cells. Cyclic GMP causes smooth muscle relaxation and increased blood flows into the corpus cavernosum. The inhibition of phosphodiesterase type 5 (PDE5) by sildenafil enhances erectile function by increasing the amount of cGMP $[1,2]$.

To investigate the single-dose bioequivalence of test 2; Satenafil 100 mg Film-Coated Tablets manufactured by Organo for Pharmaceutical and Chemical Industries (Organo Pharma). For Helwan pharmaceutical (Sildenafil $100 \mathrm{mg}$ ) and VIAGRA $100 \mathrm{mg}$ Film-Coated Tablets manufactured by Pfizer Egypt S.A.E., Cairo, A.R.E. under License of Pfizer -Inc., USA and its Subsidiary in the UK (Sildenafil $100 \mathrm{mg}$ ) given to healthy adult males under fasting conditions. For the ln-transformed ratio (test product/reference product) for the bioequivalence parameters $\left(\mathrm{C}_{\max }, \mathrm{AUC}_{0-\mathrm{t}}\right.$, and $\left.\mathrm{AUC}_{0-\infty}\right)$ while other pharmacokinetic parameters of $\mathrm{K}_{\mathrm{e}}, \mathrm{t}_{1 / 2}, \mathrm{~T}_{\max }$, and $\left(\mathrm{AUC}_{\mathrm{t}} / \mathrm{AUC}_{\infty}\right) \%$ were reported. The influence of sequence, product, and period effect were tested by ANOVA $[3,4]$. Since drug formulation plays a key role in drug absorption, variations are expected from one formula to another for the same particular drug. Moreover, drug pharmacodynamics can be affected by its pharmacokinetics, which is invariably influenced by drug product formulation. All these necessitate the need for a biometric tool to prove the drug pharmaceutical equivalence or bioequivalence. Accordingly, the interchangeable use of bioequivalent products is justified and should afford the same therapeutic efficacy $[5,6]$.

\section{Ethics considerations}

This research will be carried out in accordance with conditions stipulated by international clinical research guidelines and the principles enunciated in the Declaration of Helsinki resolved in Helsinki in 1964s and amended in Scotland, 2000; and the ICH harmonized tripartite guideline regarding Good Clinical Practice (GCP) adopted by the European Agency for the Evaluation of Medicinal Products. In addition, all local regulatory requirements will be adhered to, in particular, those which afford greater ion to the study of the participants (15) (Table 1).

\begin{tabular}{|l|l|l|l|l|l|l|l|l|}
\hline No & Initials & *Gender & $\begin{array}{l}\text { Age } \\
(\mathbf{y e a r s})\end{array}$ & $\begin{array}{l}\text { Weight } \\
\mathbf{( k g )}\end{array}$ & Height $(\mathbf{m})$ & **BMI $\left(\mathbf{k g} / \mathbf{m}^{2}\right)$ & *** Smoking & **** Pregnancy \\
\hline 1 & HMA & M & 42 & 79 & 1.69 & 28 & MS & NA \\
\hline 2 & MAA & M & 37 & 89 & 1.7 & 30.8 & NS & NA \\
\hline 3 & SMI & M & 35 & 94 & 1.75 & 31 & NS & NA \\
\hline
\end{tabular}


Citation: Eliwa HA, Eldin AA, Salah MM (2018) Pharmacological Study of Sildenafil on Healthy Volunteers. J Clin Exp Pharmacol 8: 256. doi: 10.4172/2161-1459.1000256

Page 2 of 10

\begin{tabular}{|c|c|c|c|c|c|c|c|c|c|c|}
\hline 4 & $|A|$ & \multicolumn{2}{|l|}{ M } & 90 & 1.7 & 31.1 & MS & \multicolumn{3}{|l|}{ NA } \\
\hline 5 & HZA & \multicolumn{2}{|l|}{ M } & 59 & 1.66 & 21.4 & MS & \multicolumn{3}{|l|}{ NA } \\
\hline 6 & $\mathrm{MSH}$ & \multicolumn{2}{|l|}{ M } & 65 & 1.64 & 24 & MS & \multicolumn{3}{|l|}{ NA } \\
\hline 7 & AES & \multicolumn{2}{|l|}{ M } & 87 & 1.64 & 29 & NS & \multicolumn{3}{|l|}{ NA } \\
\hline 8 & $\mathrm{AHA}$ & \multicolumn{2}{|l|}{ M } & 59 & 1.68 & 21 & MS & \multicolumn{3}{|l|}{ NA } \\
\hline 9 & MHM & \multicolumn{2}{|l|}{ M } & 70 & 1.77 & 22.4 & MS & \multicolumn{3}{|l|}{ NA } \\
\hline 10 & SAM & \multicolumn{2}{|l|}{ M } & 52 & 1.64 & 19 & MS & \multicolumn{3}{|l|}{ NA } \\
\hline 11 & SSA & \multicolumn{2}{|l|}{ M } & 84 & 1.78 & 27 & NS & \multicolumn{3}{|l|}{ NA } \\
\hline 12 & IES & \multicolumn{2}{|l|}{ M } & 98 & 1.76 & 32 & NS & \multicolumn{3}{|l|}{ NA } \\
\hline 13 & HMA & \multicolumn{2}{|l|}{ M } & 68 & 1.85 & 20 & MS & \multicolumn{3}{|l|}{ NA } \\
\hline 14 & MAM & \multicolumn{2}{|l|}{ M } & 55 & 1.73 & 18 & MS & \multicolumn{3}{|l|}{ NA } \\
\hline 15 & MHA & \multicolumn{2}{|l|}{ M } & 59 & 1.63 & 22 & MS & \multicolumn{3}{|l|}{ NA } \\
\hline 16 & SFM & \multicolumn{2}{|l|}{ M } & 52 & 1.7 & 18 & MS & \multicolumn{3}{|l|}{ NA } \\
\hline 17 & FMS & $M$ & 18 & 80 & 1.72 & 27 & MS & NA & & \\
\hline 18 & MAT & $M$ & 21 & 97 & 1.7 & 33.4 & MS & NA & & \\
\hline "M-male, & emale & & & & & & & & & \\
\hline${ }^{* *} \mathrm{BMI}$ is $\mathrm{E}$ & Mass Ind & range 18 & 0 inclusiv & $\mathrm{g} / \mathrm{m} 2)$ & & & & & & \\
\hline${ }^{* \star *}$ NS-No & noker, MS & derate S & er (less th & igarettes & day) & & & & & \\
\hline${ }^{* * * *}$ NA-N & Applicable & & & & & & & & & \\
\hline Physical & amination & & & & & & & & & \\
\hline $\begin{array}{l}\text { Subject } \\
\text { No. }\end{array}$ & Subject & Kidney & Spine & Lymph & Extremities & Neurological and & Genitalia & Rectum & Mental & Others \\
\hline & Initial & & & & & & & & & \\
\hline 1 & HMA & $\mathrm{N}^{*}$ & $\mathrm{~N}$ & $\mathrm{~N}$ & $\mathrm{~N}$ & $N$ & $\mathrm{~N}$ & $\mathrm{~N}$ & $\mathrm{~N}$ & $\mathrm{~N}$ \\
\hline 2 & MAA & N & N & N & $\mathrm{N}$ & $\mathrm{N}$ & N & $\mathrm{N}$ & N & $\mathrm{N}$ \\
\hline 3 & SMI & N & N & N & N & N & N & $\mathrm{N}$ & N & $\mathrm{N}$ \\
\hline 4 & $|A|$ & $\mathrm{N}$ & $\mathrm{N}$ & $\mathrm{N}$ & $\mathrm{N}$ & $\mathrm{N}$ & $\mathrm{N}$ & $\mathrm{N}$ & $\mathrm{N}$ & $\mathrm{N}$ \\
\hline 5 & HZA & N & N & N & N & N & N & $\mathrm{N}$ & N & $\mathrm{N}$ \\
\hline 6 & $\mathrm{MSH}$ & $\mathrm{N}$ & $\mathrm{N}$ & $\mathrm{N}$ & $\mathrm{N}$ & $\mathrm{N}$ & $\mathrm{N}$ & $\mathrm{N}$ & $\mathrm{N}$ & $\mathrm{N}$ \\
\hline 7 & AES & N & $\mathrm{N}$ & N & N & N & N & $\mathrm{N}$ & N & $\mathrm{N}$ \\
\hline 8 & $\mathrm{AHA}$ & N & $N$ & N & N & N & N & $\mathrm{N}$ & N & $\mathrm{N}$ \\
\hline 9 & MHM & N & N & N & N & $\mathrm{N}$ & N & $\mathrm{N}$ & N & $\mathrm{N}$ \\
\hline 10 & SAM & $\mathrm{N}$ & $\mathrm{N}$ & $\mathrm{N}$ & $\mathrm{N}$ & $\mathrm{N}$ & $\mathrm{N}$ & $\mathrm{N}$ & $\mathrm{N}$ & $\mathrm{N}$ \\
\hline 11 & SSA & $\mathrm{N}$ & $\mathrm{N}$ & $\mathrm{N}$ & $\mathrm{N}$ & $\mathrm{N}$ & $\mathrm{N}$ & $\mathrm{N}$ & $\mathrm{N}$ & $\mathrm{N}$ \\
\hline 12 & IES & $\mathrm{N}$ & $\mathrm{N}$ & $\mathrm{N}$ & $\mathrm{N}$ & $\mathrm{N}$ & $\mathrm{N}$ & $\mathrm{N}$ & $\mathrm{N}$ & $\mathrm{N}$ \\
\hline 13 & HMA & $\mathrm{N}$ & $\mathrm{N}$ & $\mathrm{N}$ & $\mathrm{N}$ & $N$ & $\mathrm{~N}$ & $\mathrm{~N}$ & $\mathrm{~N}$ & $\mathrm{~N}$ \\
\hline 14 & MAM & $\mathrm{N}$ & $\mathrm{N}$ & $\mathrm{N}$ & $\mathrm{N}$ & $\mathrm{N}$ & $\mathrm{N}$ & $\mathrm{N}$ & $\mathrm{N}$ & $\mathrm{N}$ \\
\hline
\end{tabular}


Citation: Eliwa HA, Eldin AA, Salah MM (2018) Pharmacological Study of Sildenafil on Healthy Volunteers. J Clin Exp Pharmacol 8: 256. doi:

\begin{tabular}{|l|l|l|l|l|l|l|l|l|l|l|}
\hline 15 & MHA & N & N & N & N & N & N & N & N & N \\
\hline 16 & SFM & N & N & N & N & N & N & N & N & N \\
\hline 17 & FMS & N & N & N & N & N & N & N & N & N \\
\hline 18 & MAT & N & N & N & N & N & N & N & N & N \\
\hline * N- Normal results
\end{tabular}

Table 1: Demographic characteristics and physical examination.

\section{Inclusion criteria}

Sex: Male

Age range between 18 and 55 years

Body Mass Index (BMI) of 18-30 (inclusive both) $\mathrm{kg} / \mathrm{m} 2$ with a minimum of $50 \mathrm{~kg}$ weight.

The absence of evidence of any clinically significant deviation from the normal medical condition. Medical history and physical examination is performed within three weeks preceding the study start date.

Laboratory test results are within the normal ranges or deviation is dismissed as clinically-insignificant by the clinical investigator and the principal investigator. Laboratory tests are performed within six months preceding the study start date.

No history of allergy to the active or inactive ingredients in the products.

Volunteer willing to adhere to the protocol requirements and to provide written informed consent.

\section{Methods and Procedures}

Study Drug Administration: On study day 1 of each study period, the study drugs were administered according to a randomization plan.

Treatment 1: One Film-Coated Tablets Test 1 Sildenamax (Sildenafil $100 \mathrm{mg}$ ) taken with $240 \mathrm{~mL}$ of water (measured with a $100 \mathrm{~mL}$ cylinder) at room temperature $[7,8]$.
Treatment 2: One Film-Coated Tablets Test 2 Satenafil (Sildenafil $100 \mathrm{mg}$ ) taken with $240 \mathrm{~mL}$ of water (measured with a $100-\mathrm{mL}$ cylinder) at room temperature.

Treatment 3: One Film-Coated Tablets VIAGRA ${ }^{\oplus}$ (Sildenafil 100 $\mathrm{mg}$ ) taken with $240 \mathrm{~mL}$ of water (measured with a $100-\mathrm{mL}$ cylinder) at room temperature [9].

\section{Bio-analytical drug determination methodology}

In this study, a high-performance liquid chromatography (HPLC) method coupled with a mass spectrometer was used for analysis of Sildenafil in plasma and the results were satisfactory. The method was validated for selectivity, specificity, linearity, precision and accuracy, recovery, stability and dilution integrity according to USFDA guidelines (supplementary file) $[10,11]$

A high-performance liquid chromatographic (Shimadzu Prominence with rack changer) method coupled with mass spectrometric detection (LC-MS/MS(API4000)) was developed, optimized and validated at MRC laboratories for the determination of Sildenafil in human plasma. The method was fully validated according to the "FDA Bioanalytical Method Validation Guidelines 2003". The linearity of the assay method was verified within the concentration range of $25-2000 \mathrm{ng} / \mathrm{ml}$. All results were within the acceptance criteria as stated in the recommended guidelines. The mean recovery of Sildenafil was $87.65 \%$ at $75 \mathrm{ng} / \mathrm{mL}$, and $77.95 \%$ at $1500 \mathrm{ng} / \mathrm{ml}$. The described method is proved to be sensitive, accurate and reproducible with a lower limit of quantification of $25 \mathrm{ng} / \mathrm{mL}$ for Sildenafil (Table 2) $[12,13]$.

\begin{tabular}{|l|l|}
\hline Item & Conditions \\
\hline Column & x terra MS C18 $4.5^{\star} 50 \mathrm{~mm}, 3.5 \mu \mathrm{m}$ \\
\hline Column Temperature & Room temperature \\
\hline Injection Volume & $50 \mu \mathrm{l}$ \\
\hline Mobile Phase & $70 \%$ Acetonitrile HPLC grade, 20\% Ammonium formate $0.1 \mathrm{~N}, 10 \%$ formic acid $0.1 \%$ \\
\hline Flow Rate & $0.55 \mathrm{ml} / \mathrm{min}$ \\
\hline Detector & MS/MS \\
\hline Internal Standard (IS) & Esomeprazole \\
\hline
\end{tabular}

Table 2: Chromatographic conditions and parameters. 
Citation: Eliwa HA, Eldin AA, Salah MM (2018) Pharmacological Study of Sildenafil on Healthy Volunteers. J Clin Exp Pharmacol 8: 256. doi:

Page 4 of 10

\section{Mass spectrometric conditions}

The mass spectrometer was an API 4000+HPLC Prominence. The ion polarity was set in positive mode, and the source was Turbolon spray. The nebulizer gas was air (zero grade) and Nitrogen was the auxiliary, curtain, and collision gas (Tables 3 and 4).

\begin{tabular}{|l|l|l|l|l|l|l|}
\hline Compound & Q1 Mass (Da) & Q3 Mass (Da) & CE (volts) & CXP (volts) & EP (volts) & DP (volts) \\
\hline Esomeprazole (IS) & 346.2 & 198.2 & 16.24 & 15 & 10 & 31.01 \\
\hline Sildenafil & 474.9 & 100.3 & 40.28 & 17.86 & 10 & 127.96 \\
\hline
\end{tabular}

Table 3: Mass spectrometric conditions.

\begin{tabular}{|l|l|}
\hline Instrument & Prominence HPLC Shimadzu connected to Mass spectrometer API 4000, MDS Sciex, Toronto, Canada \\
\hline Analytical Balance & BOECO BB1-31, 4-d, Germany, pre-use regular calibration was done daily. \\
\hline Vortex & VWR VV3 S540 International West Charter-USA. \\
\hline Centrifuge & Changsha Xiangzhi Instruments, Hunan, China \\
\hline Vacuum Pump & TID-15, Apparao Garden, Choolai, Chennai, India. \\
\hline pH meter & $\begin{array}{l}\text { Corning pH meter, UK, the pre-application celebrative adjustment was done daily using standardized buffers of different pH-values } \\
(4.00-11.00) .\end{array}$ \\
\hline
\end{tabular}

Table 4: List of equipment used.

\section{Statistical analysis}

Statistical analysis was performed using a Kinetica version 5.1 (Thermo Scientific, USA).

The chemicals, reagents, and Standards used in the present study, are displayed as follows:

Sildenafil working standard

Esomeprazole working standard

Water for chromatography (Sharlau, Spain)

Acetonitrile, HPLC grade (Sigma Aldrich Chemie GmbH, Steinheim-Germany)

Ammonium Formate, Dichloromethane, (Sigma Aldrich Chemie $\mathrm{GmbH}$, Steinheim-Germany)

Blank plasma obtained from the Holding Company for Biological Products \& Vaccines (VACSERA), Giza, Egypt [14].

\section{Statistical Results}

Oral administration of Test 1; Sildenamax (Sildenafil $100 \mathrm{mg}$ ) and Test 2; Satenafil (Sildenafil $100 \mathrm{mg}$ ) to healthy adult volunteers at different interval time (0-24), as compared by the reference drug VIAGRA (Sildenafil $100 \mathrm{mg}$ ) to healthy adult volunteers at different interval time (0-24) showed that:

1. The rate and extent of absorption of Sildenafil through measurement of Sildenafil from, Satenafil $100 \mathrm{mg}$ Film-Coated Tablets manufactured and VIAGRA $100 \mathrm{mg}$.

2. The Test product, Satenafil (Sildenafil $100 \mathrm{mg}$ ) is bioequivalent to the reference drug VIAGRA ${ }^{\circ}$ (Sildenafil $100 \mathrm{mg}$ ).
Therefore, the data obtained in this study prove, by appropriate statistical methods, the essential similarity of plasma levels of Sildenafil from the test product Satenafil $100 \mathrm{mg}$ and from the reference product VIAGRA $100 \mathrm{mg}$ suggesting the equal clinical efficacy of these two products. The product, Satenafil $100 \mathrm{mg}$ Film-Coated Tablets by Organo for Pharmaceutical and Chemical Industries (Organo Pharma) for Helwan pharmaceutical, may be used interchangeably with the reference product VIAGRA ${ }^{\circ} 100 \mathrm{mg}$ Tablets by Pfizer Egypt. That was shown the tested product has an acceptable therapeutic efficacy (Tables 5-18 and Figures 1-3) [15].

\begin{tabular}{|l|l|l|l|l|l|l|}
\hline & $\mathbf{0}$ & $\mathbf{2}$ & $\mathbf{4}$ & $\mathbf{6}$ & $\mathbf{1 2}$ & $\mathbf{2 4}$ \\
\hline Average & 5.987133 & 346.6 & 134.7346 & 91.44 & 23.257 & 9.953833 \\
\hline STDEV & 26.93952 & 213.1998 & 64.91686 & 62.50886 & 18.93988 & 28.67496 \\
\hline
\end{tabular}

Table 5: Plasma concentration levels of Sildenafil ( $\mathrm{ng} / \mathrm{mL}$ ) following administration of a single oral dose of treatment (2) test product Satenafil $100 \mathrm{mg}$.

\begin{tabular}{|l|l|l|l|l|l|l|}
\hline & $\mathbf{0}$ & $\mathbf{2}$ & $\mathbf{4}$ & $\mathbf{6}$ & $\mathbf{1 2}$ & $\mathbf{2 4}$ \\
\hline Average & 7.920182 & 314.3067 & 130.1533 & 78.04333 & 21.75333 & 1.400167 \\
\hline STDEV & 34.31167 & 147.4286 & 56.7114 & 39.54986 & 15.85234 & 1.906898 \\
\hline
\end{tabular}

Table 6: Plasma concentration levels of Sildenafil $(\mathrm{ng} / \mathrm{mL})$ following administration of a single oral dose of Treatment (3) reference product VIAGRA $100 \mathrm{mg}$. 
Citation: Eliwa HA, Eldin AA, Salah MM (2018) Pharmacological Study of Sildenafil on Healthy Volunteers. J Clin Exp Pharmacol 8: 256. doi:

Page 5 of 10

\begin{tabular}{|c|c|c|c|c|c|c|c|c|c|}
\hline Vol no. & Period & $C_{\max }(\mathbf{n g} / \mathbf{m L})$ & $\mathbf{T}_{\max }(\mathbf{h})$ & $A \cup C_{0-t}(n g \cdot h / m L)$ & $A U C_{E x t}(n g \cdot h / m L)$ & $\mathrm{AUC}_{0-\infty}(\mathrm{ng} \cdot \mathrm{h} / \mathrm{mL})$ & $\% \mathrm{AUC}_{\mathrm{Ext}}$ & $K_{e}(h-1)$ & $t_{1 / 2}(h)$ \\
\hline 1 & 1 & 1120 & 1.5 & 1572.85 & 190.572 & 1763.42 & 10.807 & 0.1627 & 4.2605 \\
\hline 2 & 1 & 660 & 1.25 & 1481.32 & 144.745 & 1626.06 & 8.9015 & 0.1934 & 3.5844 \\
\hline 3 & ॥ & 903 & 0.75 & 895.717 & 73.4119 & 969.129 & 7.5751 & 0.4461 & 1.5538 \\
\hline 4 & I & 1060 & 1.5 & 1745.43 & 20.9461 & 1766.38 & 1.1858 & 0.1757 & 3.9442 \\
\hline 5 & III & 447 & 2.5 & 1481.63 & 132.759 & 1614.38 & 8.2235 & 0.2164 & 3.2026 \\
\hline 6 & III & 645 & 1.25 & 1439.18 & 6.9103 & 1446.09 & 0.4779 & 0.194 & 3.5723 \\
\hline 7 & III & 702 & 1.25 & 2933.62 & 47.1775 & 2980.8 & 1.5827 & 0.1584 & 4.376 \\
\hline 8 & ॥ & 606 & 1.75 & 1228.02 & 213.055 & 1441.08 & 14.784 & 0.2399 & 2.8899 \\
\hline 9 & III & 1000 & 2.5 & 3211 & 225.493 & 3436.49 & 6.5617 & 0.1055 & 6.5705 \\
\hline 10 & III & 770 & 2.5 & 1719.41 & 23.3652 & 1742.77 & 1.3407 & 0.1643 & 4.2197 \\
\hline 11 & III & 509 & 1.25 & 1008.72 & 8.2229 & 1016.94 & 0.8086 & 0.4071 & 1.7027 \\
\hline 12 & I & 778 & 1.5 & 1106.96 & 41.3787 & 1148.34 & 3.6033 & 0.2694 & 2.5729 \\
\hline 13 & 1 & 580 & 0.75 & 1093.32 & 12.9729 & 1106.29 & 1.1727 & 0.3736 & 1.8552 \\
\hline 14 & II & 580 & 1.5 & 1331.2 & 37.1899 & 1368.39 & 2.7178 & 0.3292 & 2.1057 \\
\hline 15 & ॥ & 861 & 2 & 2721.47 & 13.9401 & 2735.42 & 0.5096 & 0.214 & 3.2384 \\
\hline 16 & 1 & 421 & 1.25 & 1901.16 & 0.0739 & 1901.23 & 0.0039 & 0.4047 & 1.7129 \\
\hline 17 & $\|$ & 831 & 2 & 2140.53 & 35.8445 & 2176.38 & 1.647 & 0.3508 & 1.9761 \\
\hline 18 & 1 & 567 & 0.75 & 1097.68 & 7.6605 & 1105.34 & 0.693 & 0.2135 & 3.2464 \\
\hline 19 & III & 1180 & 0.25 & 2457.13 & 11.112 & 2468.24 & 0.4502 & 0.2239 & 3.0951 \\
\hline 20 & 1 & 905 & 1.75 & 1455.93 & 60.0267 & 1515.95 & 3.9597 & 0.2886 & 2.4015 \\
\hline 21 & $\|$ & 459 & 2.5 & 1253.85 & 79.2279 & 1333.08 & 5.9432 & 0.2092 & 3.314 \\
\hline 22 & III & 300 & 1.25 & 1101.85 & 73.1319 & 1174.98 & 6.2241 & 0.25 & 2.7729 \\
\hline 23 & ॥ & 451 & 1 & 1435.22 & 176.859 & 1612.08 & 10.971 & 0.1823 & 3.8017 \\
\hline 24 & $\|$ & 414 & 0.75 & 1281.55 & 2.3033 & 1283.85 & 0.1794 & 0.2528 & 2.7418 \\
\hline 25 & III & 372 & 0.5 & 865.9 & 40.2234 & 906.123 & 4.4391 & 0.2508 & 2.7641 \\
\hline 26 & II & 360 & 0.5 & 847.342 & 42.3274 & 889.669 & 4.7577 & 0.245 & 2.8292 \\
\hline 27 & 1 & 989 & 3 & 1608.8 & 166.496 & 1775.29 & 9.3785 & 0.1949 & 3.5559 \\
\hline 28 & $\|$ & 594 & 0.25 & 2521.65 & 67.8498 & 2589.5 & 2.6202 & 0.137 & 5.0603 \\
\hline 29 & I & 615 & 1 & 1877.6 & 4.933 & 1882.53 & 0.262 & 0.2405 & 2.8816 \\
\hline 30 & III & 418 & 0.16667 & 2266.3 & 414.66 & 2680.96 & 15.467 & 0.0848 & 8.1702 \\
\hline \multicolumn{2}{|l|}{ Average } & 669.9 & 1.3556 & 1636.078 & 79.1623 & 1715.239 & 4.5749 & 0.2393 & 3.3324 \\
\hline \multicolumn{2}{|l|}{ STDEV } & 245.046 & 0.7492 & 632.5037 & 93.0308 & 658.0727 & 4.4464 & 0.0885 & 1.4083 \\
\hline \multicolumn{2}{|l|}{ CV\% } & 36.5794 & 55.2674 & 38.6598 & 117.5191 & 38.3662 & 97.191 & 36.996 & 42.261 \\
\hline
\end{tabular}

Table 7: Pharmacokinetic parameters of Sildenafil following administration of single oral dose of Treatment (2) test product Satenafil 100 mg to 30 volunteers. 
Citation: Eliwa HA, Eldin AA, Salah MM (2018) Pharmacological Study of Sildenafil on Healthy Volunteers. J Clin Exp Pharmacol 8: 256. doi:

Page 6 of 10

\begin{tabular}{|c|c|c|c|c|c|c|c|c|c|}
\hline SI no. & Period & $\mathrm{C}_{\max }(\mathrm{ng} / \mathrm{mL})$ & $\mathbf{T}_{\max }(\mathrm{h})$ & $A \cup C_{0-t}(n g . h / m L)$ & $\begin{array}{l}\mathrm{AUC}_{\mathrm{Ext}} \\
\text { (ng.h/mL) }\end{array}$ & $\mathrm{AUC}_{0-\infty}(\mathrm{ng} \cdot \mathrm{h} / \mathrm{mL})$ & $\% \mathrm{AUC}_{\text {Ext }}$ & $K_{e}(h-1)$ & $\mathbf{t}_{1 / 2}(\mathrm{~h})$ \\
\hline 1 & ॥ & 515 & 2.5 & 1909.58 & 106.39 & 2015.96 & 5.2774 & 0.2469 & 2.8078 \\
\hline 2 & III & 495 & 2.5 & 1472.25 & 123.186 & 1595.44 & 7.7211 & 0.2229 & 3.1096 \\
\hline 3 & III & 606 & 1.25 & 1190.05 & 79.9184 & 1269.97 & 6.293 & 0.3579 & 1.9368 \\
\hline 4 & III & 774 & 0.5 & 1946.35 & 84.8929 & 2031.24 & 4.1794 & 0.2686 & 2.5803 \\
\hline 5 & ॥ & 508 & 1 & 1649.01 & 7.6936 & 1656.7 & 0.4644 & 0.2158 & 3.212 \\
\hline 6 & 1 & 615 & 1.25 & 1449.6 & 8.6482 & 1458.25 & 0.5931 & 0.1851 & 3.7442 \\
\hline 7 & 1 & 649 & 2 & 2363.52 & 8.389 & 2371.9 & 0.3537 & 0.2476 & 2.7998 \\
\hline 8 & III & 769 & 1 & 1810.54 & 45.1567 & 1855.7 & 2.4334 & 0.3276 & 2.1158 \\
\hline 9 & II & 501 & 1 & 1621.18 & 0.1068 & 1621.29 & 0.0066 & 0.4092 & 1.6937 \\
\hline 10 & 1 & 487 & 2 & 1074.2 & 7.4244 & 1081.63 & 0.6864 & 0.4948 & 1.4009 \\
\hline 11 & $\|$ & 396 & 0.75 & 552.493 & 28.0789 & 580.572 & 4.8364 & 0.3798 & 1.825 \\
\hline 12 & III & 483 & 0.75 & 898.315 & 19.4081 & 917.723 & 2.1148 & 0.3031 & 2.2869 \\
\hline 13 & $\|$ & 923 & 0.5 & 2007.56 & 15.5152 & 2023.08 & 0.7669 & 0.4343 & 1.5961 \\
\hline 14 & 1 & 527 & 0.75 & 859.933 & 8.9968 & 868.93 & 1.0354 & 0.3941 & 1.7588 \\
\hline 15 & 1 & 559 & 1.25 & 1784.62 & 73.332 & 1857.96 & 3.9469 & 0.2408 & 2.8781 \\
\hline 16 & ॥ & 621 & 1.75 & 1152.73 & 25.3576 & 1178.09 & 2.1524 & 0.3288 & 2.108 \\
\hline 17 & I & 819 & 2 & 2763.83 & 1.6873 & 2765.52 & 0.061 & 0.3087 & 2.2457 \\
\hline 18 & III & 549 & 1.25 & 1623.91 & 16.2142 & 1640.12 & 0.9886 & 0.1912 & 3.6252 \\
\hline 19 & ॥ & 428 & 0.75 & 1233.71 & 29.2673 & 1262.98 & 2.3173 & 0.1497 & 4.63 \\
\hline 20 & ॥ & 890 & 1.5 & 2507.91 & 433.555 & 2941.47 & 14.7394 & 0.0834 & 8.3117 \\
\hline 21 & III & 393 & 1.5 & 922.326 & 45.6588 & 967.985 & 4.7169 & 0.2756 & 2.5151 \\
\hline 22 & 1 & 326 & 1.25 & 1047.25 & 92.8824 & 1140.13 & 8.1466 & 0.1997 & 3.4705 \\
\hline 23 & I & 344 & 0.25 & 1134.03 & 189.067 & 1323.1 & 14.2897 & 0.1679 & 4.1294 \\
\hline 24 & III & 412 & 2 & 1395.34 & 19.4311 & 1414.77 & 1.3735 & 0.1805 & 3.84 \\
\hline 25 & $\|$ & 925 & 2.5 & 1205.23 & 16.7845 & 1222.02 & 1.3735 & 0.4082 & 1.6983 \\
\hline 26 & III & 1210 & 1.25 & 1278.77 & 25.7824 & 1304.55 & 1.9763 & 0.3039 & 2.2806 \\
\hline 27 & II & 1200 & 0.25 & 2356.96 & 7.2764 & 2364.24 & 0.3078 & 0.2394 & 2.895 \\
\hline 28 & I & 453 & 0.25 & 1600.44 & 1.5901 & 1602.03 & 0.0993 & 0.32 & 2.1662 \\
\hline 29 & III & 983 & 1.75 & 1899.75 & 1.1175 & 1900.87 & 0.0588 & 0.3168 & 2.188 \\
\hline 30 & I & 912 & 0.08333 & 1081.62 & 41.8158 & 1123.43 & 3.7222 & 0.2742 & 2.5283 \\
\hline \multicolumn{2}{|c|}{ Average } & 642.4 & 1.2444 & 1526.4336 & 52.1541 & 1578.5883 & 3.2344 & 0.2825 & 2.8126 \\
\hline \multicolumn{2}{|c|}{ STDEV } & 242.8532 & 0.7006 & 532.8043 & 84.4805 & 557.0102 & 3.8308 & 0.0939 & 1.3165 \\
\hline \multicolumn{2}{|l|}{ CV\% } & 37.80405 & 56.2998 & 34.9052 & 161.9823 & 35.2853 & 118.4382 & 33.2382 & 46.8091 \\
\hline
\end{tabular}

Table 8: Pharmacokinetic parameters of Sildenafil following administration of single oral dose of Treatment (3) reference product VIAGRA 100 mg to 30 volunteers. 
Citation: Eliwa HA, Eldin AA, Salah MM (2018) Pharmacological Study of Sildenafil on Healthy Volunteers. J Clin Exp Pharmacol 8: 256. doi:

Page 7 of 10

\begin{tabular}{|c|c|c|c|c|}
\hline \multirow[t]{2}{*}{ Time (h) } & \multicolumn{2}{|c|}{ Satenafil 100 mg Film-Coated Film-Coated Tablets } & \multicolumn{2}{|c|}{ VIAGRA ${ }^{\circledR} 100 \mathrm{mg}$ Film-Coated Tablets } \\
\hline & Average & $\pm S D$ & Average & $\pm \mathrm{SD}$ \\
\hline 0 & 5.9871 & 26.9395 & 6.00841 & 30.609 \\
\hline 0.833 & 11.3803 & 33.394 & 36.96 & 170.579 \\
\hline 0.1667 & 58.0473 & 123.692 & 64.323 & 171.756 \\
\hline 0.25 & 148.843 & 280.405 & 136.267 & 257.398 \\
\hline 0.5 & 225.485 & 200.295 & 210.371 & 229.818 \\
\hline 0.75 & 323.766 & 237.422 & 272.251 & 224.026 \\
\hline 1 & 339.608 & 167.76 & 329.797 & 180.167 \\
\hline 1.25 & 307.678 & 182.145 & 376.785 & 239.068 \\
\hline 1.5 & 383.393 & 245.775 & 367.133 & 192.092 \\
\hline 1.75 & 343.133 & 190.298 & 320.99 & 176.741 \\
\hline 2 & 346.6 & 216.845 & 314.307 & 149.949 \\
\hline 2.5 & 299.803 & 199.334 & 272.04 & 187.269 \\
\hline 3 & 228.02 & 193.341 & 211.21 & 131.08 \\
\hline 4 & 132.136 & 64.7913 & 130.153 & 57.6809 \\
\hline 6 & 91.44 & 63.5775 & 78.0433 & 40.226 \\
\hline 8 & 45.992 & 29.6938 & 44.771 & 25.6878 \\
\hline 12 & 23.257 & 19.2637 & 21.7533 & 16.1233 \\
\hline 24 & 9.9538 & 29.1652 & 2.5546 & 8.71318 \\
\hline
\end{tabular}

Table 9: Plasma concentration Average \pm SD ( $\mathrm{ng} / \mathrm{mL}$ ) of Sildenafil following oral administration of Treatment (2) test product Satenafil $100 \mathrm{mg}$ and Treatment (3) reference product VIAGRA $100 \mathrm{mg}$ to 30 volunteers.

\begin{tabular}{|c|c|c|c|c|c|c|}
\hline & \multicolumn{3}{|l|}{ Test (B) } & \multicolumn{3}{|c|}{ Reference (C) } \\
\hline & Average & SD & CV\% & Average & SD & CV\% \\
\hline$C_{\max }(n g / m L)$ & 669.9 & $\begin{array}{l}245.045 \\
6\end{array}$ & 36.5794 & 642.4 & $\begin{array}{l}242.853 \\
2\end{array}$ & $\begin{array}{l}37.80405 \\
4\end{array}$ \\
\hline $\begin{array}{l}\mathrm{AUC}_{0-\mathrm{t}} \\
\text { (ng.h/mL) }\end{array}$ & $\begin{array}{l}1636.07 \\
8\end{array}$ & $\begin{array}{l}632.503 \\
7\end{array}$ & 38.6598 & $\begin{array}{l}1526.433 \\
6\end{array}$ & $\begin{array}{l}532.804 \\
3\end{array}$ & 34.9052 \\
\hline $\begin{array}{l}{ }^{A U C} C_{\text {Ext }} \\
\text { (ng.h/mL) }\end{array}$ & 79.1623 & 93.0308 & $\begin{array}{l}117.519 \\
1\end{array}$ & 52.1541 & 84.4805 & 161.9823 \\
\hline $\begin{array}{l}\mathrm{AUC}_{0-\infty} \\
\text { (ng.h/mL) }\end{array}$ & $\begin{array}{l}1715.23 \\
94\end{array}$ & $\begin{array}{l}658.072 \\
7\end{array}$ & 38.3662 & $\begin{array}{l}1578.588 \\
3\end{array}$ & $\begin{array}{l}557.010 \\
2\end{array}$ & 35.2853 \\
\hline $\begin{array}{l}\mathrm{AUC}_{\mathrm{Ext}} / \\
\mathrm{AUC} \mathrm{C}_{0-\infty}\end{array}$ & 4.5749 & 4.4464 & 97.1908 & 3.2344 & 3.8308 & 118.4382 \\
\hline
\end{tabular}

\begin{tabular}{|l|l|l|l|l|l|l|}
\hline $\mathrm{K}_{\mathrm{e}}(\mathrm{h}-1)$ & 0.2393 & 0.0885 & 36.996 & 0.2825 & 0.0939 & 33.2382 \\
\hline $\mathrm{t}_{1 / 2}(\mathrm{~h})$ & 3.3324 & 1.4083 & 42.261 & 2.8126 & 1.3165 & 46.8091 \\
\hline $\mathrm{T}_{\max }(\mathrm{h})$ & 1.3556 & 0.7492 & 55.2674 & 1.2444 & 0.7006 & 56.29983 \\
\hline
\end{tabular}

Table 10: Mean values of the pharmacokinetic parameters of Sildenafil derived from the plasma concentration-time profiles of treatment (2) test product Satenafil $100 \mathrm{mg}$ and treatment (3) reference Product VIAGRA $^{\circ} 100 \mathrm{mg}$ following single oral administration of $100 \mathrm{mg}$ Sildenafil to 30 healthy volunteers.

\begin{tabular}{|l|l|l|l|}
\hline & Point Estimate & Lower Confidence Limit & Upper Confidence Limit \\
\hline $\mathrm{C}_{\max }(\mathrm{ng} / \mathrm{mL})$ & $104.15 \%$ & $90.08 \%$ & $120.42 \%$ \\
\hline $\mathrm{AUC}_{0-\mathrm{t}}(\mathrm{ng} \cdot \mathrm{h} / \mathrm{mL})$ & $106.59 \%$ & $95.12 \%$ & $119.44 \%$ \\
\hline
\end{tabular}


Citation: Eliwa HA, Eldin AA, Salah MM (2018) Pharmacological Study of Sildenafil on Healthy Volunteers. J Clin Exp Pharmacol 8: 256. doi:

Table 11: $90 \%$ Confidence interval and point estimate for $\mathrm{C}_{\max }, \mathrm{AUC}_{0-\mathrm{t}} \& \mathrm{AUC}_{0-\infty}$.

\begin{tabular}{|l|l|}
\hline & $\mathrm{CV}_{\text {intra }}$ \\
\hline $\mathrm{C}_{\max }(\mathrm{ng} / \mathrm{mL})$ & 0.052487 \\
\hline $\mathrm{AUC}_{0-\mathrm{t}}(\mathrm{ng} \cdot \mathrm{h} / \mathrm{mL})$ & 0.036135 \\
\hline $\mathrm{AUC}_{0-\infty}(\mathrm{ng} . \mathrm{h} / \mathrm{mL})$ & 0.036143 \\
\hline
\end{tabular}

\begin{tabular}{|l|l|l|l|l|}
\hline Error & 56 & 6.3245 & 0.11294 & \multirow{2}{*}{} \\
\cline { 1 - 4 } Total & $\mathbf{8 9}$ & $\mathbf{1 0 . 1 3 7}$ & \\
\cline { 1 - 3 } & & &
\end{tabular}

Table 13: ANOVA table with confidence interval for $\operatorname{Ln} C_{\text {max }}$.

Table 12: Intra-subject variability for $\mathrm{C}_{\max }, \mathrm{AUC}_{0-\mathrm{t}} \& \mathrm{AUC}_{0-\infty}$.

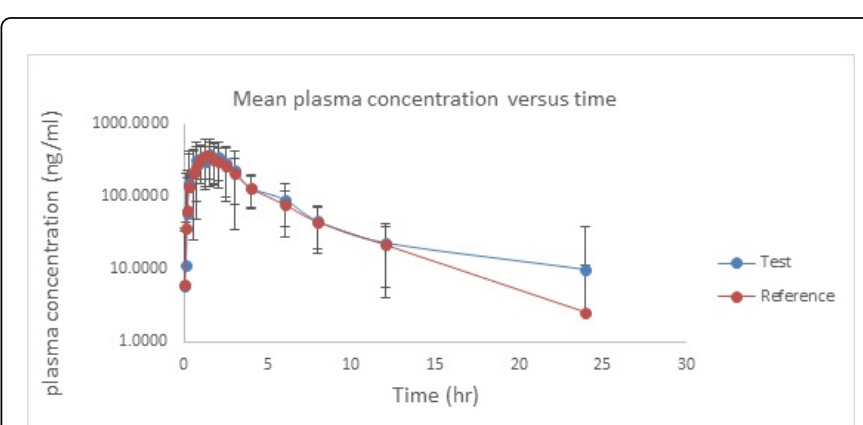

\begin{tabular}{|l|l|l|l|l|l|l|}
\hline Source & $\begin{array}{l}\text { D } \\
\text { F }\end{array}$ & SS & MS & F-Ratio & p-value & Significance \\
\hline Period & 2 & 0.2718 & 0.1359 & 1.928 & 0.1549 & Non-significant \\
\hline $\begin{array}{l}\text { Subject } \\
\text { (Sequence) }\end{array}$ & 29 & 6.1129 & 0.21079 & 2.991 & $\begin{array}{l}0.000217 \\
3\end{array}$ & Significant \\
\hline Treatment & 2 & $\begin{array}{l}0.09284 \\
9\end{array}$ & $\begin{array}{l}0.04642 \\
4\end{array}$ & 0.6587 & 0.5215 & Non-significant \\
\cline { 1 - 3 } Error & 56 & 3.9468 & $\begin{array}{l}0.07047 \\
9\end{array}$ & & & \\
\cline { 1 - 3 } Total & 89 & 10.424 & \multicolumn{2}{|l}{} & \\
\cline { 1 - 3 } & & & & & &
\end{tabular}

Figure 1: Mean plasma concentration vs time profile for Sildenafil after administration of an oral single-dose of $100 \mathrm{mg}$ Sildenafil of the test product (Satenafil $100 \mathrm{mg}$ ) and the reference product (VIAGRA $100 \mathrm{mg}$ ).

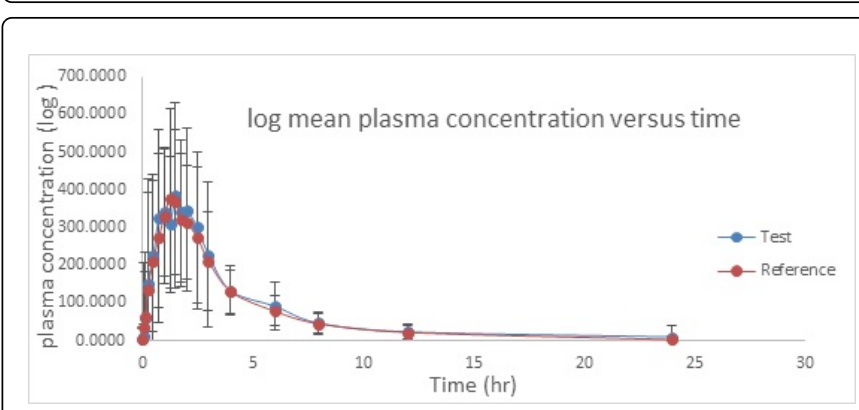

Figure 2: Logarithmic mean plasma concentration vs time profile for Sildenafil after administration of an oral single-dose of $100 \mathrm{mg}$ Sildenafil of the test product (Satenafil $100 \mathrm{mg}$ ) and the reference product (VIAGRA $100 \mathrm{mg}$ ).

\begin{tabular}{|l|l|l|l|l|l|l|}
\hline Source & DF & SS & MS & $\begin{array}{l}\text { F- } \\
\text { Ratio }\end{array}$ & $\begin{array}{l}\mathbf{p}- \\
\text { value }\end{array}$ & Significance \\
\hline Period & 2 & $\begin{array}{l}0.01517 \\
3\end{array}$ & $\begin{array}{l}0.00758 \\
7\end{array}$ & $\begin{array}{l}0.0671 \\
8\end{array}$ & 0.9351 & $\begin{array}{l}\text { Non- } \\
\text { significant }\end{array}$ \\
\hline $\begin{array}{l}\text { Subject } \\
\text { (Sequence) }\end{array}$ & 29 & 3.7113 & 0.12798 & 1.133 & 0.337 & $\begin{array}{l}\text { Non- } \\
\text { significant }\end{array}$ \\
\hline Treatment & 2 & $\begin{array}{l}0.08626 \\
4\end{array}$ & $\begin{array}{l}0.04313 \\
2\end{array}$ & 0.3819 & 0.6843 & $\begin{array}{l}\text { Non- } \\
\text { significant }\end{array}$ \\
\hline
\end{tabular}

Table 14: ANOVA table with confidence interval for $\mathrm{Ln} \mathrm{AUC}_{\mathrm{t}}$.

\begin{tabular}{|c|c|c|c|c|c|c|}
\hline Source & $\begin{array}{l}D \\
F\end{array}$ & ss & MS & F-Ratio & p-value & Significance \\
\hline Period & 2 & $\begin{array}{l}0.2261 \\
6\end{array}$ & 0.11308 & 1.627 & 0.2056 & Non-significant \\
\hline $\begin{array}{l}\text { Subject } \\
\text { (Sequence) }\end{array}$ & 29 & 6.0934 & 0.21012 & 3.024 & $\begin{array}{l}0.000190 \\
4\end{array}$ & Significant \\
\hline Treatment & 2 & $\begin{array}{l}0.0677 \\
8\end{array}$ & 0.03389 & 0.4877 & 0.6166 & Non-significant \\
\hline Error & 56 & 3.8917 & $\begin{array}{l}0.06949 \\
5\end{array}$ & & & \\
\hline Total & 89 & 10.279 & & & & \\
\hline
\end{tabular}

Table 15: ANOVA table with confidence interval for $\operatorname{Ln} \mathrm{AUC}_{\text {inf. }}$

\begin{tabular}{|c|c|c|c|c|c|c|}
\hline Source & DF & SS & MS & F-Ratio & $\begin{array}{l}p- \\
\text { value }\end{array}$ & Significance \\
\hline Period & 2 & $\begin{array}{l}0.5574 \\
1\end{array}$ & 0.2787 & 0.5651 & 0.5715 & Non-significant \\
\hline $\begin{array}{l}\text { Subject } \\
\text { (Sequence) }\end{array}$ & 29 & 12.045 & $\begin{array}{l}0.4153 \\
5\end{array}$ & 0.8421 & 0.6874 & Non-significant \\
\hline Treatment & 2 & $\begin{array}{l}0.9199 \\
1\end{array}$ & $\begin{array}{l}0.4599 \\
5\end{array}$ & 0.9326 & 0.3996 & Non-significant \\
\hline Error & 56 & 27.62 & $\begin{array}{l}0.4932 \\
1\end{array}$ & & & \\
\hline Total & 89 & 41.142 & & & & \\
\hline
\end{tabular}

Table 16: ANOVA table with confidence interval for $\mathrm{T}_{\max }$. 
Citation: Eliwa HA, Eldin AA, Salah MM (2018) Pharmacological Study of Sildenafil on Healthy Volunteers. J Clin Exp Pharmacol 8: 256. doi:

Page 9 of 10

\begin{tabular}{|c|c|c|c|c|c|c|c|}
\hline Conc. \% & Conc. $(\mu \mathrm{g} / \mathrm{mL})$ & Peak Area 1 & Peak Area 2 & Peak Area 3 & Average Peak Area & SD & RSD (\%) \\
\hline 25 & 25 & 262 & 262.1 & 264.8 & 262.95 & 1.59 & 0.60 \\
\hline 50 & 50 & 522.1 & 526.5 & 526.3 & 524.94 & 2.48 & 0.47 \\
\hline 75 & 75 & 806 & 808 & 811.9 & 808.64 & 3.04 & 0.38 \\
\hline 100 & 100 & 1074.5 & 1075 & 1083.2 & 1077.56 & 4.88 & 0.45 \\
\hline 125 & 125 & 1366.6 & 1363.4 & 1360.9 & 1363.64 & 2.81 & 0.21 \\
\hline 150 & 150 & 1650.1 & 1639.2 & 1639.4 & 1644.67 & 7.7 & 0.47 \\
\hline 200 & 200 & 2152.2 & 2158.1 & 2162.7 & 2157.66 & 5.24 & 0.24 \\
\hline
\end{tabular}

Table 17: Average peak area response for different concentrations of sildenafil.

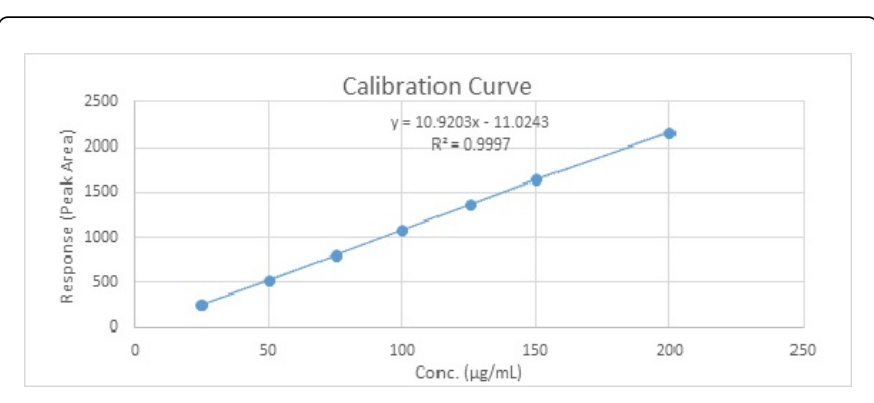

Figure 3: Response peak area versus concentration in $\mu \mathrm{g} / \mathrm{ml}$ for calibration curve of test Sildenafil.

\begin{tabular}{|l|l|}
\hline Regression line equation & $\mathrm{y}=\mathrm{ax}+\mathrm{b}$ \\
\hline Slope, $\mathrm{a}$ & 10.92 \\
\hline Intercept, b & -11.024 \\
\hline $\mathrm{R}^{2}$ & 0.9997 \\
\hline LOD $(\mu \mathrm{g} / \mathrm{mL})$ & 1.197 \\
\hline LOQ $(\mu \mathrm{g} / \mathrm{mL})$ & 3.628 \\
\hline LOD $($ Limit of Detection) $=$ & $3.3 \times \delta$ (standard deviation)/Slope \\
\hline LOQ (Limit of Quantitation) $=$ & $10 \times \delta$ (standard deviation)/Slope \\
\hline
\end{tabular}

Table 18: Results and stastistics.

\section{Discussion}

In this study, Satenafil $100 \mathrm{mg}$ Film-Coated Film Coated Tablets, Batch no.:T30046 (Exp. date: 04/2018), manufactured by Organo for Pharmaceutical and Chemical Industries (Organo Pharma) for Helwan pharmaceutical, Egypt is bioequivalent to the reference product VIAGRA 100 mg Film-Coated Tablets, Batch No.: 6702 (Exp. date: 01/2018) manufactured by Pfizer Egypt (Table 19).

\begin{tabular}{|l|l|}
\hline $90.08 \%-120.42 \%(104.15 \%)$ & for $C_{\max }$ \\
\hline $96.41 \%-121.25 \%(108.12 \%)$ & for $\mathrm{AUC}_{\mathrm{t}}$ \\
\hline
\end{tabular}

$95.12 \%-119.44 \%(106.59 \%)$ for $A \cup C_{\text {inf }}$

Table 19: With an estimated 90\% confidence interval (point estimate).

The present results showed that the two products are interchangeable and they deliver equivalent amounts of Sildenafil to the systemic circulation. In the current study, oral administration of Satenafil Film-Coated Tablets (Sildenafil $100 \mathrm{mg}$ ) to healthy adult volunteers were assessed for their ability to improve erectile dysfunction as same as VIAGRA Film-Coated Tablets (Sildenafil 100 mg) manufactured by Pfizer Egypt.

\section{Conclusion}

Bioequivalence could be demonstrated for Sildenafil within the prescribed $90 \%$ confidence interval of $80.00 \%$ to $125.00 \%$ for $\mathrm{AUC}_{0-\mathrm{t}}$ and $\mathrm{AUC}_{0-\infty}$ and for $\mathrm{C}_{\max }$ to be within $80.00 \%$ to $125.00 \%$ with respect to the parametric method on Ln-transformed data.

The test product, Satenafil $100 \mathrm{mg}$ Film-Coated Tablets by Organo for Pharmaceutical and Chemical Industries (Organo Pharma), investigated in this study was shown to be bioequivalent with the reference product; VIAGRA $100 \mathrm{mg}$ Film-Coated Tablets by Pfizer Egypt and its Subsidiary in the UK. Plasma levels may be used as surrogate parameters for therapeutic response. Therefore, the data obtained in this study prove, by appropriate statistical methods, the essential similarity of plasma levels of Sildenafil from the test product Satenafil $100 \mathrm{mg}$ Film-Coated Tablets and from the reference product VIAGRA $^{*} 100 \mathrm{mg}$ Film-Coated Tablets suggesting the equal clinical efficacy of these two products. The product, Satenafil $100 \mathrm{mg}$ FilmCoated Tablets by Organo, may be used interchangeably with the reference product VIAGRA ${ }^{\circ} 100 \mathrm{mg}$ Film-Coated Tablets by Pfizer Egypt. That was shown the tested product has an acceptable therapeutic efficacy.

\section{References}

1. Radicioni M, Castiglioni C, Giori A, Cupone I, Frangione V, et al. (2017) Bioequivalence study of a new sildenafil $100 \mathrm{mg}$ orodispersible film compared to the conventional film-coated $100 \mathrm{mg}$ tablet administered to healthy male volunteers. Drug Des Devel Ther 11: 1183-1192.

2. Wang D, Li Y, Xu L, Pan SM, Li XM, et al. (2016) A single dose, randomized, open-label, cross-over bioequivalence study of sildenafil citrate tablets in healthy Chinese volunteers. Int J Clin Pharmacol Ther 55: 186-193. 
Citation: Eliwa HA, Eldin AA, Salah MM (2018) Pharmacological Study of Sildenafil on Healthy Volunteers. J Clin Exp Pharmacol 8: 256. doi:

Page 10 of 10

3. Gao X, Ndongo M, Checchio N, Cook TM, Duncan J, et al. (2015) A randomized, open-label 3-way crossover study to investigate the relative bioavailability and bioequivalence of crushed sildenafil $20 \mathrm{mg}$ tablets mixed with apple sauce, extemporaneously prepared suspension (EP), and intact sildenafil $20 \mathrm{mg}$ tablets in healthy volunteers under fasting conditions. Clin Pharmacol Drug Dev 4: 74-80.

4. Bate R, Mathur A, Lever H, Thakur M, Graedon D, et al. (2016) Generics substitution, bioequivalence standards, and international oversight: Complex issues facing the FDA. Trends Pharmacol Sci 37: 184-191.

5. Traynor K (2010) FDA mulls changes to bioequivalence standards. Am J Health Syst Pharm 67: 864.

6. Spinola A, Almeida C, Filipe S, Tanguay A, Yritia M (2008) Bioequivalence study of two tablet formulations of sildenafil. Arzneimittelforschung 58: 122-125.

7. Remane Y, Leopold CS (2006) Transfer of the experimental methodology described in the FDA guidance for corticosteroid bioequivalence testing to pharmacodynamic effects caused by nicotinates. J Cosmet Dermatol 5: 289-293.

8. Fox GN. (2005) Levothyroxine and FDA bioequivalence ratings. J Fam Pract 54: 179.

9. Mandal U, Musmade P, Chakraborty M, Rajan DS, Chakravarti M, et al. (2004) Bioequivalence study of sildenafil citrate tablets in healthy human volunteers. Boll Chim Farm 143: 345-349.
10. Hen ML, Shah V, Patnaik R, Adams W, Hussain A, et al. (2001) Bioavailability and bioequivalence: an FDA regulatory overview. Pharm Res 18: 1645-1650.

11. Williams RL, Patnaik RN, Chen ML (2000) The basis for individual bioequivalence. FDA population and individual bioequivalence working group. Eur J Drug Metab Pharmacokinet 25: 13-17.

12. Kimanani E, Stypinski D, Curtis G, Stiles M, Heessels P, et al. (2000) A contract research organization's response to the new FDA guidances for bioequivalence/bioavailability studies for orally administered drug products. J Clin Pharmacol 40: 1102-1108.

13. Patnaik RN, Lesko LJ, Chen ML, Williams RL (1997) Individual bioequivalence. New concepts in the statistical assessment of bioequivalence metrics. FDA Group. Clin Pharmacokinet 33: 1-6.

14. Hauck WW, Chen ML, Hyslop T, Patnaik R, Schuirmann D, et al. (1996). Mean difference vs variability reduction: tradeoffs in aggregate measures for individual bioequivalence. FDA Individual Bioequivalence Working Group. Int J Clin Pharmacol Ther 34: 535-541.

15. World medical association declaration of helsinki-ethical principles for medical research involving human subjects. Adopted by the 18th WMA General Assembly Helsinki, Finland, June 1964 and amended by the 59th WMA General Assembly, Seoul. 